\title{
A Study of Patients With Normal Coronary Angiogram Over A Period of 14 Months in A Military Hospital
}

\author{
MOHAMMAD ABDUL MALEK ${ }^{1}$, S M MAMUNUR RAHMAN ${ }^{1}$, SYED ASIF IQBAL ${ }^{1}$, COL ZEHAD KHAN ${ }^{1}$, \\ MUKHLESUR RAHMAN ${ }^{2}$, SYEDA ALEYA SULTANA ${ }^{1}$, WALI-UR RAHMAN ${ }^{1}$, AKM MOHIUDDIN BHUIYAN ${ }^{3}$ \\ ${ }^{1}$ Department of Cardiology, Combined Military Hospital, Dhaka Cantonment, Dhaka, ${ }^{2}$ Department of Cardiology, Bangabandhu \\ Sheikh Mujib Medical University, Dhaka, ${ }^{3}$ Department of Cardiology, National Institute of Ophthalmology, Dhaka \\ Address for Crrespondance: Dr (Major) Mohammad Abdul Malek, ${ }^{1}$ Department of Cardiology, Combined Military Hospital, \\ Dhaka.E mail: malek100932@yahoo.com
}

\begin{abstract}
:
Objectives: The objectives were to study the incidence of the normal coronary artery anatomy, to determine their clinical, electrocardiographic, stress testing, echocardiographic and angiographic characteristics.

Methods: This is a retrospective study carried out in cardiac center, Combined Military Hospital, Dhaka, Bangladesh on patients seen during the period from November 2010 to Dec 2011. The coronary artery angiography data was collected by a check list and was revised by the authors.

Results: Twelve hundred and two fifty patients who had coronary angiography which showed normal coronary artery or minor coronary artery disease were included in this study. $13 \%$ were females. The mean age of the patients was 45.6. 74\% patients had one or more coronary risk factors and predominant risk factor was hypertension (31\%). More than 50\% patients had significant cardiac symptoms and about $40 \%$ study subjects were found to have prominent ECG changes which preclude noninvasive tests. Only $38 \%$ of study subjects underwent exercise ECG tests, among them 12\% had positive ETT. Two hundred and forty nine patients (20\%) had normal coronary artery anatomy. Right dominant circulation was found in $79 \%$ of the patients.

Conclusions: The incidence of normal coronary artery anatomy is similar to those reported in other literatures. Although there are limitations of common noninvasive tests to exclude coronary artery disease, proper clinical evaluation and appropriate use of other noninvasive investigations will definitely reduce the burden of coronary angiogram in patients with angina or suspected ischemic heart disease.
\end{abstract}

Keywords: angina, coronary artery disease, electrocardiography (ECG), treadmill test (ETT), coronary angiography (CAG).

\section{Introduction:}

Despite careful patient selection, we do come across in the cardiac catheterization laboratory patients with normal or near normal coronary angiograms (CAG). This not only adds to ever increasing health costs, it also subjects the patient to an invasive test with its small risk of morbidity and mortality. Over the period from November 2010 to December 2011, we did 1687 cases including pediatrics catheterization in catheterization laboratory, CMH Dhaka. Among all coronary angiogram done in 1250 cases we noted a total of 249 patients with normal coronary artery or minor coronary artery disease (defined as lesions causing a coronary luminal diameter reduction less than 30\%). We were interested in these groups of patients in regards to their clinical and investigational characteristics. We hoped to identify the factors that could account for their symptoms or abnormal test result.

\section{Material and methods}

\section{Study design}

This is a cross sectional, single center, retrospective study conducted in Combined Military Hospital (CMH), Dhaka. We studied all consecutive patients who had coronary angiogram over a 14 months period from November 2010 to December 2011. We traced the coronary angiogram reports of these 1250 patients and identified 249 with normal or near normal coronary artery disease. A normal coronary angiogram was defined as one with the absence of any visible angiographic signs of atherosclerosis, thrombosis or spontaneous spasm. We then went through the case notes of these 249 patients to check on their clinical data and reviewed the electrocardiographic, treadmill and echocardiographic results.

\section{Data analysis}

Data retrieved from hospital notes included age, sex, trade of the patients, diagnosis, reason for coronary angiogram, 
presence of cardiac risk factors, result of preangiography exercise test(where applicable), echocardiographic findings and important angiographic result. Study patients included both entitled (military, civilian entitled, family members of the entitled and retired patients) and nonentitled which included civilian non entitled (CNE) and civilian patients. Entitled patients are eligible for treatment in CMH. CNE patients are parents and in laws of military personnel. Diagnosis at catheterization laboratory was based on initial presentation. Unstable angina (UA) and acute myocardial infarction (AMI) were diagnosed by relevant history along with supportive ECG and cardiac biomarkers following standard criteria. Old myocardial infarction was labelled by related ECG evidence with or without supportive clinical features and echocardiographic findings. Chronic ischemic heart disease (IHD) was diagnosed mainly by strong clinical evidence or if patient was on treatment for IHD. Observation for ischemic heart disease (Obsn for IHD) was made if there are atypical symptoms in presence of one or more major coronary risk factors with or without supportive ECG and other investigations. Chest pain was the diagnosis where there was other evidence to suspect ischemic heart disease but non invasive test failed to exclude coronary artery disease. Consultant cardiologist reviewed the indication of CAG in all cases during the precath check up but indication of CAG was not uniform all the times because CMH, Dhaka is the only tertiary care referral military hospital in Bangladesh with all cardiac facilities. For the sake of standard fitness in armed forces, threshold for coronary angiography was sometimes low in case of serving patients, especially to exclude coronary artery disease. Five important major coronary risk factors were considered in the study. Risk factors were diabetes mellitus, hypertension, smoking, family history of coronary artery disease and dyslipidemia.

\section{Electrocardiogram, Echocardiogram and Treadmill tests}

ECG done nearest to the coronary angiography date was reviewed for each patient. Significant ST/T changes (deep $\mathrm{T}$ inversion in anterior and inferior leads or ST depression), ST elevation myocardial infarction (STEMI), Pathological Q, Left ventricular hypertrophy (LVH) which fulfills voltage criteria, and other important ECG finding were noted. Others ECG included Left bundle branch block (LBBB), Right bundle branch block (RBBB) and Complete heart block. Treadmill reports where applicable, were reviewed with regards to the degree and type of ST changes. In case where the test was done more than six month prior to the CAG date, or if treadmill was not done at all, these were labeled as 'not done'.

\section{Coronary angiography}

Coronary angiography was carried out via the percutaneous femoral approach using standard Judkin's technique. Six French catheters were used in all cases. Angiograms of coronary arteries were obtained in multiple views with varying degree of cranial and caudal tilting depending on the segment of the artery being imaged. GE innova2000 equipment with $\mathrm{C}$ arm was used for CAG. Left ventricugram (where applicable) was done with 6 French pigtail catheters.

\section{Statistical analysis}

Continuous data are presented as mean \pm SD and ranged when appropriate. Continuous variables were compared by Simple Pearson's correlation \& Student's t-test. Chisquare analysis was used for categorical data. A two-tailed p value $<0.05$ was defined statistically significant. The software SPSS version 17.0 (SPSS Inc., Chicago, Illinois) was used for statistical analysis.

\section{Result:}

Clinical characteristics of 249 patients at angiography were shown in table 1.

\begin{tabular}{lcc}
\hline Character & Number & percent \\
\hline Patients & 249 & 100 \\
Age(years) & & \\
$\quad$ Mean & 45.6 & \\
$\quad$ Range & $18-75$ & \\
Gender & & \\
$\quad$ Male & 206 & 83 \\
$\quad$ Female & 43 & 17 \\
Symptoms & & \\
Typical angina & 38 & 15 \\
Atypical angina & 65 & 26 \\
$\quad$ Non anginal pain & 35 & 14 \\
Breathlessness & 30 & 12 \\
$\quad$ Asymptomatic & 34 & 14 \\
Exercise ECG & & \\
Positive & 29 & 12 \\
Equivocal & 19 & 8 \\
Negative & 45 & 18 \\
$\quad$ Not done & 156 & 62 \\
Echocardiography & & \\
Normal & 147 & 59 \\
RWMA & 26 & 10 \\
HCM & 19 & 8 \\
VHD & 28 & 11 \\
LVEF $<55 \%$ & 22 & 8 \\
Others & 28 & 11 \\
\hline
\end{tabular}


Values presented are number (\%) of subjects; RWMA, Regional wall motion abnormality; HCM, Hypertrophic cardiomyopathy; VHD, Valvular heart disease; LVEF, left ventricular ejection fraction.

The mean age of the patients was $45.6 \pm 10.5$ years. There were a total of 206 (83\%) male and 43 (17\%) females. 136 (54\%) patients had age between 36 years to 50 years and only 4 (2\%) patients had age more than 65 years. Among all patients, 180 (72\%) were entitled and rest 69(28\%) were nonentitled which included CNE patients (7\%) also (Fig 2).

We considered five major coronary risk factors (Diabetes mellitus, Hypertension, Dyslipidemia, Smoking and Family history) in all patients. Predominant risk factors were hypertension $77(31 \%)$ and $31(12 \%)$ patients had DM (Fig 3). 90(36\%) patients had no risk factors, maximum 112 (46\%) patients had single risk factor and only one patient had four coronary risk factors.

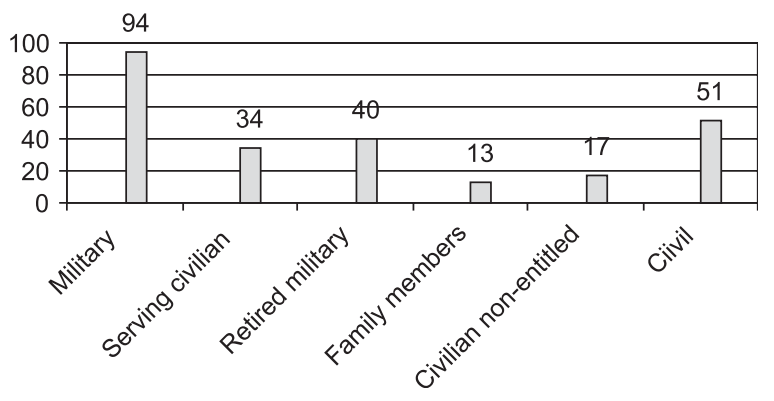

Fig.-1: Trade of the patients

We defined 'elevated cholesterol' as those whose total serum cholesterol was $>5.2 \mathrm{mmol} / \mathrm{l}$ and LDL cholesterol was $>3 \mathrm{mmol} / \mathrm{l}$. Smokers are patients who were engaged in cigarette smoking at the time of study when they were admitted for coronary angiography. Patients who were labeled as hypertensive or diabetics must be diagnosed by qualified physicians and have been advised for treatment. Family history of coronary artery disease refers to those whose parent or siblings have been diagnosed to have coronary artery disease at age $<55$ years.

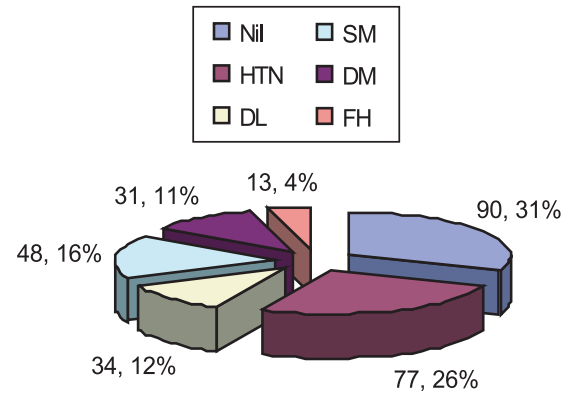

Data expressed as number.
Thirty eight (15\%) patients had typical angina, sixty five (26\%) had atypical angina and thirty (12\%) patients had exertional dyspnea. Patients with VHD and Shunt anomaly mainly presented with breathlessness. Thirty four (14\%) patients had no symptoms but they had significant ECG changes at rest(Fig 3).

Acute myocardial infarction was precatheterization diagnosis in14 (6\%) patients and 5(2\%) patients were labelled as old MI where reanalyzed coronary artery was found during angiography (Fig 4). Data expressed as number (percent)
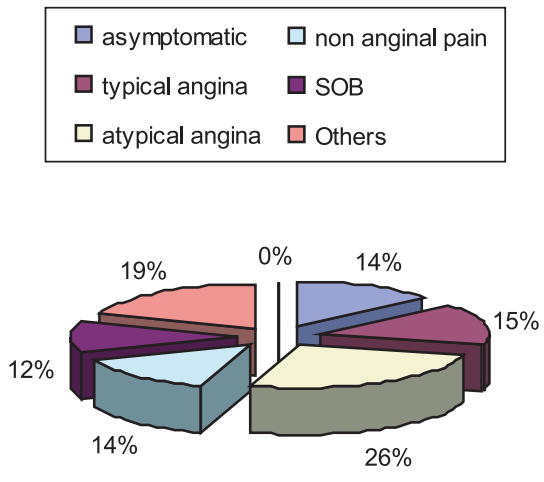

Data expressed as number (percent)

16(6\%) patients had $Q$ wave myocardial infarction in routine ECG, 100(40\%) patients had significant deep T inversion in multiple leads and 79 (32\%) subjects had normal ECG(Table 2). 31(12\%) patients had valvular heart disease where CAG was done as preoperative requirement.

Among all patients, exercise tolerance test was done in 93(38\%) patients, of those 30 (12\%) patients had positive ETT, 19(8\%) had equivocal response to exercise. ETT was not done in majority of the patients (74\%) because either ETT was not indicated or they had absolute or relative contraindication (Table 1).

Table-II

ECG changes of the patients

\begin{tabular}{lcc}
\hline ECG changes & Frequency & Percent \\
\hline Normal ECG & 80 & 32 \\
Significant ST/T changes & 27 & 10.8 \\
LVH & 12 & 4.8 \\
AMI & 4 & 1.6 \\
OMI & 12 & 4.8 \\
Non specific ST/T changes & 56 & 22.4 \\
Others & 59 & 23.7 \\
\hline
\end{tabular}

26(10\%) patients had regional wall motion abnormality with impaired left ventricular function and rest of the 
patients had normal left ventricular function. 19(8\%) patients had typical morphological features suggestive of hypertrophic cardiomyopathy.

201 (80\%) of the 249 patients had completely normal coronary arteries at angiogram. 24(9\%) patients had minor CAD,10 (4\%) patients had variable coronary ectasia, $2 \%$ patients had anomalous coronary arteries and another 2\% had sluggish (TIMI2) flow despite normal CAG(Fig 5).

\begin{tabular}{|ll|}
\hline$\square$ STEMI & $\square$ OBS FOR IHD \\
$\square$ OMI & $\square$ CHEST PAIN \\
$\square$ UA/NSTEMI & $\square$ VHD \\
$\square$ Chr IHD & $\square$ OTHERS \\
\hline
\end{tabular}

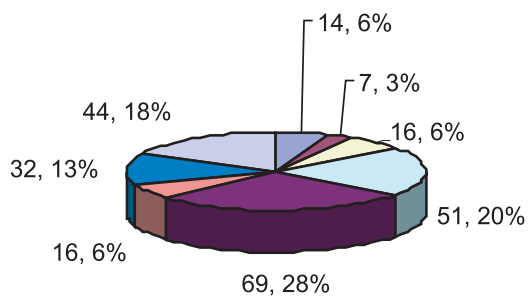

Data expressed as percent

115(46\%) patients had a large distal anterior descending (LAD) artery or type 3 (defined as distal LAD arteries that are large in caliber, and which supply the distal portion of the posterior septum), 95(38\%) medium or type 2 (defined as medium sized arteries which also supply the LV apex and 39 small or type 1 distal LAD artery( defined as LAD arteries that do not go around the cardiac apex or diminutive in size).
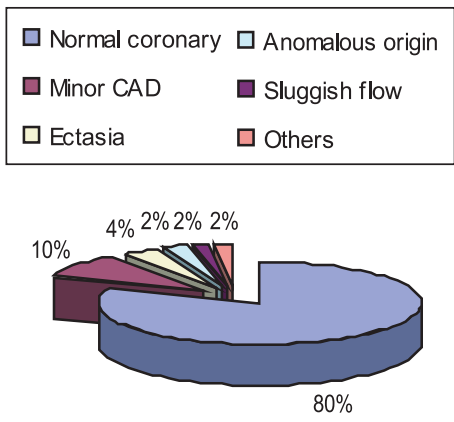

Data expressed as percent

\section{Discussion:}

The pathophysiology of angina pectoris is usually related to myocardial ischemia secondary to significant obstruction of the large epicardial coronary arteries. Occasionally patients who presents with typical angina or angina like symptoms have entirely normal or near normal coronary arteries during coronary angiography ${ }^{1,2,3}$. This combination has been descriptively known as Syndrome X. It is also known that these patients may exhibit an abnormal ST response during treadmill testing, and this often has been labeled as a 'false positive response'.

In our study mean age of the patients is $45.6 \pm 10.5 y e a r s$. This is akin to kemp's series ${ }^{4}$, where the average age was 47 years. Coronary artery disease is more prevalent in males which are consistent in our study. Most patients (97\%) in our study have one or more coronary risk factors. In our study, most prevalent coronary risk factor was hypertension which correlates well with another large epidemiological study ${ }^{5}$ where individuals with hypertension or diabetes showed a higher risk of CHD. This study revealed that about two third of study patients had significant symptoms and 20\% patients had positive/ equivocal exercise ETT.

One important possible etiology for normal coronaries of these large patients group is hypertension ${ }^{6}$ which is predominant risk factor in this study. Others possible explanation for angina with normal coronary are microvascular disease, cardiac syndrome $\mathrm{X}$, variant angina and hypertrophic cardiomyopathy ${ }^{3,4,6}$. So, magnetic resonance imaging and gated-single photon emission computed tomography (SPECT), intravascular ultrasound (IVUS), coronary flow reserve and endothelial function testing may help in further risk assessment of this group of patients ${ }^{7}$.

68\% patients had significant abnormal resting ECG which included patients with HCM, hypertension and valvular heart diaease. Male patients, middle age, high prevalence of coronary risk factor, significant ST-T changes (which preclude exercise ETT ) and valvular heart disease - all these features may be biased; the presence of these features probably influenced their selection for subsequent angiography.

In our study 29(20\%) patients had positive and another 19(8\%) patients had indeterminate ETT response. Among all positive cases 26(90\%) patients were male but incidence of false positive ETT is more common among female 8,9 . This is possibly due to large number of these group patients with hypertension, baseline ECG changes and LVH. O Cannon et $\mathrm{al}^{10}$ have done extensive work in these patients and suggested that the underlying pathophysiology is that of an abnormal vasodilator response of small vessel (vessels ranging from 50-500 microns in diameter) are too small to be detected in routine coronary angiography. Also, a normal CAG does 
not exclude ischemia due to these small vessels. Hence, these patients' stress test findings are strictly speaking not 'false positive' for myocardial ischemia but rather 'false positive' for major epicardial stenosis ${ }^{11}$. In fact, exercise thallaium-201 scans have been shown to produce 'false positive' result in these group of patients as well. However, these patients have benign prognosis generally, and their symptoms may be relieved by medications ${ }^{12}$.

In this study, incidence of normal coronary angiogram is high (20\%) which is different from another similar study ${ }^{6}$ where incidence is $11 \%$ but in our study we included patients with STEMI, Old MI, Hypertrophic cardiomyopathy and valvular heart disase which comprised about $29 \%$ of study patients. In another study ${ }^{13}$ of acute chest pain with suspected STEMI, incidence of normal coronary was $2.6 \%$ where most of the patients were basically misdiagnosed because those patients were suffering from myocarditis, cardiomyopathy or pulmonary embolism. A. Da Costa et al ${ }^{14}$ described different etiology like spasm, embolism, oral contraceptive pill in their study with STEMI with normal coronary angiogram.

We noted interesting features during analysis of the coronary angiogram-14(30\%) of those patients with positive or equivocal ETT had type 1 left anterior descending artery which was found in another study ${ }^{6}$. (This information is routinely coded during reporting of coronary angiogram). This does suggest that the small distal LAD artery in patients with chest pain, positive ETT and normal CAG might not just be a chance observation but there could exist a possible underlying association but we did not perform left ventricular end diastolic pressure in patients with normal CAG. Bush et $\mathrm{al}^{15}$ noted in an earlier study that patient with elevated LVEDP may have anginal chest pain with angiographically normal coronary arteries. Myocardial infarction with 'normal' coronary arteries (MINCA) typically occurs in the under-50s ${ }^{16,17,18}$. Usually there is no history of angina or previous myocardial infarction (MI), and risk factors for ischemic heart disease (IHD) may be absent ${ }^{19}$ but the most likely underlying disorder in myocardial infarction with normal angiograms is coronary artery disease, which predisposes the artery to vasospasm and thrombosis. Symptoms and electrocardiographic (ECG) findings are similar to those of MI with angiographic coronary disease, though the infarct sizes tend to be smaller. The rate of post-MI complications, such as malignant arrhythmias, heart failure and hypotension, is lower and the long-term prognosis is more favourable ${ }^{20}$.
Conclusion: The presence of chest pain in patients with either normal coronary angiograms or with nonobstructive coronary artery disease (CAD) is not only a frequent clinical finding but also a clinical and therapeutic challenge. It may be caused by coronary vasomotility disorders which comprise epicardial as well as microvascular dysfunction. Traditional risk factors (such as hypertension and diabetes) as well as cardiomyopathies be associated with functional as well as structural changes which are major predisposing factors for the occurrence of both epicardial coronary vasospasm and microvascular disease, and cause the occurrence of AP in the absence of obstructive CAD

\section{References:}

1. Likoff W, Siegal BL, Kasprian H, Paradox of normal selective angiogram in patients considered to have unmistakable coronary heart disease. N Eng J Med. 1976; 276, 1063-6

2. Anoop Chauhan. Syndrome X - angina and normal coronary angiography. Postgrad MedJ 1995; 71: 341-345.

3. Ali Yilmaz, Udo Sechtem. Angina pectoris in patients with normal coronary angiograms: current pathophysiological concepts and therapeutic options. Heart 2012; 98:1020.

4. Kemp HG, Elliot WC, Gorlin R. Anginal syndrome with normal coronary arteriography. Trans Assoc Am Physicians.1967; 80:5970 .

5. Jeannette Lee, Derrick Heng, Kee Seng Chia, Suok Kai Chew, Bee Yian Tanc and Kenneth Hughesa Int. J. Epidemiol. 2001; 30 (5): 983-88.

6. Y T Lim, M H Choo, K Kon, PYan, J K Yeoh, C S Soo, et al. Areview of patients with a normal coronary angiogram over a 3 year period. Singapore MED J 1992; 33:455-59.

7. P Widimsky, B Stellova, M Aschermann, M Branny, M Zelizko, J Stasek, et al. Prevalence of normal coronary angiography in the acute phase of suspected ST-elevation myocardial infarction: Experience from the PRAGUE studies. Can J Cardiol 2006; 22(13):1147-52.

8. Faisal AW, Abid AR, Azhar M.J. Ayub Med Coll Abbottabad. Exercise Tolerance Test: a comparison between true positive and false positive test results.2007; 19(4):71-4.

9. Qayser S. Habeeb. Reliability and accuracy of exercise tolerance test in the diagnosis of coronary heart disease. DMJ 2007; 1(1): 98-104.

10. Cannon III RO, Epstein SE. "Microvascular Angina” as a cause of chest pain with angiographically normal coronary arteries. Am J Cardiol 1988; 61:1338-43.

11. Epstein SE, Cannon III RO, Bonow RO. Exercise testing in patients with microvascular angina. Circulation 1991; 83(supp III): 73-6.

12. Berger BC, Abramowitz R, Park CH, Desai AG, Madsen MT, Chung EK. Abnormal Thallium 201scan in patients with chest pain and angiographically normal coronaryarteries. Am J Cardiol 1983; 52:365-70.

13. Raffaele Bugiardini, Lina Badimon, Peter Collins, Raimund Erbel, Kim Fox, Christian Hamm, Fausto Pinto et al. "Normal” Coronary 
Angiography, and Vascular Dysfunction: Risk Assessment Strategies; PLoS Medicine.: February 2007,Volume 4, Issue 2, e12.

14. A. Da Costa, K. Isaaz, E. Faure, S. Mourot, A. Cerisier and M. LamaudClinical. Characteristics, aetiological factors and long-term prognosis of myocardial infarction with an absolutely normal coronary angiogram. Euro Heart J 2001; 22: 1459-65.

15. Bush CA, Fanning W, Kollibash AJ, Leir CV. Elevated left ventricular filling pressure as a cause of angina in patients with normal coronary arteries. Am J Cardiol 1982, 49:950 (abstract).

16. Tun A, Khan IA. Acute myocardial infarction with angiographically normal coronary arteries. Heart Lung 2000; 29:348-50.
17. B Chandrasekaran, P A S Kurbaan. Myocardial infarction with angiographically normal coronary arteries. J R Soc Med 2002; 95:398-400

18. Sharifi M, Frohlich TG, Silverman IM. Myocardial infarction with angiographically normal coronary arteries. Chest 1995; 107:36-40.

19. Khan IA, Ansari AW. Myocardial infarction in a pre-menopausal woman with angiographically normal coronary arteries. Postgrad Med J 1998; 74:671-2.

20. Raymond R, Lynch J, Underwood D, Leatherman J, Razavi M. Myocardial infarction and normal coronary arteriography: a 10 year clinical and risk analysis of 74 patients. J Am Coll Cardiol 1988; 11:471-7. 